\title{
Lifting Symmetry Breaking Constraints with Inductive Logic Programming
}

\author{
Alice Tarzariol $^{1}$, Martin Gebser ${ }^{1,2}$, Konstantin Schekotihin ${ }^{1}$ \\ ${ }^{1}$ University of Klagenfurt, Austria \\ ${ }^{2}$ Graz University of Technology, Austria \\ alice.tarzariol@aau.at,martin.gebser@aau.at,konstantin.schekotihin@aau.at
}

\begin{abstract}
Efficient omission of symmetric solution candidates is essential for combinatorial problem solving. Most of the existing approaches are instancespecific and focus on the automatic computation of Symmetry Breaking Constraints (SBCs) for each given problem instance. However, the application of such approaches to large-scale instances or advanced problem encodings might be problematic. Moreover, the computed SBCs are propositional and, therefore, can neither be meaningfully interpreted nor transferred to other instances. To overcome these limitations, we introduce a new modeloriented approach for Answer Set Programming that lifts the SBCs of small problem instances into a set of interpretable first-order constraints using the Inductive Logic Programming paradigm. Experiments demonstrate the ability of our framework to learn general constraints from instance-specific SBCs for a collection of combinatorial problems. The obtained results indicate that our approach significantly outperforms a state-of-the-art instancespecific method as well as the direct application of a solver.
\end{abstract}

\section{Introduction}

Many combinatorial problems are relatively easy to model with the current declarative programming paradigms. Nevertheless, when the size of input instances starts to grow, solving them might become infeasible because of a large number of possible solution candidates [Dodaro et al., 2016]. In many cases, these candidates are symmetric, i.e., one candidate can simply be obtained from another by renaming constants. In order to deal with large problem instances, the ability to encode Symmetry Breaking Constraints (SBCs) in a problem representation becomes an essential skill for programmers. However, the identification of symmetric solutions and the formulation of constraints that remove them is a tedious and time-consuming task. As a result, various tools emerged that avoid the computation of symmetric solutions by, for instance, automatically finding a set of SBCs using properties of permutation groups, or applying specific search methods that detect and ignore symmetric states; see [Sakallah, 2009; Walsh, 2012; Margot, 2010] for an overview.

Respective approaches can be distinguished into instancespecific and model-oriented ones. The former methods identify symmetries for a particular instance at hand by computing and adding ground SBCs to the problem representation [Puget, 2005; Cohen et al., 2006; Drescher et al., 2011]. Unfortunately, computational advantages do not carry forward to large-scale instances or advanced encodings, where instancespecific symmetry breaking often requires as much time as it takes to solve the original problem. Moreover, ground SBCs generated by instance-specific approaches are (i) not transferable, since the knowledge obtained is limited to a single instance; (ii) usually hard to interpret and comprehend; (iii) derived from permutation group generators, whose computation is itself a combinatorial problem; and ( $i v$ ) often redundant and might result in a degradation of the solving performance.

Elevated model-oriented approaches aim to find general SBCs that depend less on a particular instance. The method of Devriendt et al. [2016] uses local domain symmetries of a given first-order theory. SBCs are generated by identifying argument positions in atoms of a formula that comprise object variables defined over the same subset of a domain given in the input. As a result, the computation of lexicographical SBCs is very fast. However, the method considers each firstorder formula separately and cannot reliably remove symmetric solutions, as it requires the analysis of several formulas at once. Mears et al. [2008] compute SBCs by generating small instances of parametrized constraints programs, and then find candidate symmetries using SAUCY [Darga et al., 2004; Codenotti et al., 2013] - a graph automorphism detection tool. Next, the algorithm removes all candidate symmetries that are valid only for some of the generated examples as well as those that cannot be proven to be parametrized symmetries using heuristic graph-based techniques. This approach can be seen as a simplified learning procedure that utilizes only negative examples represented by the generated SBCs.

In this work, we introduce a novel model-oriented method for Answer Set Programming (ASP) [Lifschitz, 2019] that aims to generalize the process of discarding redundant solution candidates for instances of a target domain using Inductive Logic Programming (ILP) [Cropper et al., 2020]. The goal is to lift the SBCs of small problem instances and to obtain a set of interpretable first-order constraints. Such con- 
straints cut the search space while preserving the satisfiability of a problem for the considered instance distribution, which improves the solving performance, especially in the case of unsatisfiability. The particular contributions of our work are:

- We suggest a method to generate a training set including positive and negative examples, allowing an ILP method to learn first-order SBCs for the problem at hand.

- We define the components of an ILP learning task enabling the generation of lexicographical SBCs for ASP.

- We show how to apply our method iteratively, to revise constraints when new permutation group generators are added or more training instances become available.

- We conduct experiments on variants of the pigeon-hole problem as well as the house-configuration problem [Friedrich et al., 2011]. The obtained results show the benefits of our approach that significantly outperforms a state-of-the-art instance-specific method as well as an ASP solver without SBCs.

The structure of this paper is the following: a brief overview of the preliminaries is given in Section 2. Section 3 describes our approach, while Section 4 illustrates its implementation and specifies the components of an ILP learning task. In Section 5, we present and discuss experimental results for some combinatorial problems. Lastly, Section 6 concludes the paper and outlines directions for future work.

\section{Background}

\subsection{Answer Set Programming}

Answer Set Programming (ASP) [Lifschitz, 2019] is a declarative programming paradigm that applies non-monotonic reasoning and relies on the stable model semantics [Gelfond and Lifschitz, 1991]. Over the past decades, ASP has attracted considerable interest thanks to its elegant syntax, expressiveness, and efficient systems implementations, showing promising results in numerous domains that include, e.g., industrial, robotics, and biomedical applications [Erdem et al., 2016; Falkner et al., 2018]. We will briefly define the syntax and semantics of ASP, and refer the reader to [Gebser et al., 2012; Lifschitz, 2019] for more detailed explanations.

Syntax. An ASP program $P$ is a set of rules $r$ of the form:

$$
a_{0} \leftarrow a_{1}, \ldots, a_{m}, \text { not } a_{m+1}, \ldots, \text { not } a_{n}
$$

where not stands for default negation and $a_{i}$, for $0 \leq i \leq n$, are atoms. An atom is an expression of the form $p(\overline{\bar{t}})$, where $p$ is a predicate, $\bar{t}$ is a possibly empty vector of terms, and the predicate $\perp$ (with an empty vector of terms) represents the constant false. Each term $t$ in $\bar{t}$ is either a variable or a constant, and a literal $l$ is either an atom $a_{i}$ (positive) or its negation not $a_{i}$ (negative). The atom $a_{0}$ is the head of a rule $r$, denoted by $H(r)=a_{0}$, and the body of $r$ includes the positive or negative, respectively, body atoms $B^{+}(r)=\left\{a_{1}, \ldots, a_{m}\right\}$ and $B^{-}(r)=\left\{a_{m+1}, \ldots, a_{n}\right\}$. A rule $r$ is called a fact if $B^{+}(r) \cup B^{-}(r)=\emptyset$, and a constraint if $H(r)=\perp$.
Semantics. The semantics of an ASP program $P$ is given in terms of its ground instantiation $P_{\text {grd }}$, replacing each rule $r \in P$ with instances obtained by substituting the variables in $r$ by constants occurring in $P$. Then, an interpretation $\mathcal{I}$ is a set of (true) ground atoms occurring in $P_{\text {grd }}$ that does not contain $\perp$. An interpretation $\mathcal{I}$ satisfies a rule $r \in P_{\text {grd }}$ if $B^{+}(r) \subseteq \mathcal{I}$ and $B^{-}(r) \cap \mathcal{I}=\emptyset$ imply $H(r) \in \mathcal{I}$, and $\mathcal{I}$ is a model of $P$ if it satisfies all rules $r \in P_{\text {grd }}$. A model $\mathcal{I}$ of $P$ is stable if it is a subset-minimal model of the reduct $\left\{H(r) \leftarrow B^{+}(r) \mid r \in P_{g r d}, B^{-}(r) \cap \mathcal{I}=\emptyset\right\}$, and we denote the set of all stable models of $P$ by $A S(P)$.

\subsection{Symmetry Breaking}

Modern approaches detect symmetries of a given object by representing it, e.g., as an instance of the graph automorphism problem, ${ }^{1}$ which is solved using methods from the group theory, see [Sakallah, 2009] for an overview.

Let $X$ be a set of $n$ elements $x_{1}, \ldots, x_{n}$, then we can define a permutation of $X$ as a bijection $\phi$ that rearranges its elements. The symmetric group $G=\left\langle X_{p}, \phi\right\rangle$ is defined by the set $X_{p}$ of all possible permutations of $X$ closed under $\phi$, and its subgroups $G^{\prime}=\left\langle X_{p}^{\prime}, \phi\right\rangle$ are called permutation groups, where $X_{p}^{\prime} \subseteq X_{p}$. In cycle notation, we represent a permutation $\pi$ as a product of disjoint cycles, where each cycle $\left(\begin{array}{lllll}x_{1} & x_{2} & x_{3} & \ldots & x_{k}\end{array}\right)$ means that the element $x_{1}$ is mapped to $x_{2}, x_{2}$ to $x_{3}$ and so on, until $x_{k}$ is mapped back to $x_{1}$; the elements mapped to themselves are not contained in the cycles. Let $g \in X_{p}$ be an element of a group $G$, then $g$ is a generator for $G$ if any other element of the set $X_{p}$ can be obtained by a finite number of applications of $\phi$. A set of generators $A_{X} \subset X_{p}$ of a symmetric group $G$ generates a subgroup $G^{\prime}$ of $G$ if $G^{\prime}$ is the smallest subgroup containing $A_{X}$. If $G^{\prime}$ is all of $G$, then $A_{X}$ is a group generator. A generator is redundant if it can be expressed in terms of other generators. The goal of graph automorphism algorithms is to find a set $A_{X}$ of irredundant generators, i.e., a set that contains no redundant generators. Given a total order of the elements in $X$, an irredundant group generator can be used to introduce a set of constraints that eliminates all permutations of its elements: this approach is called lex-leader and produces Symmetry Breaking Constraints (SBCs). Considering a set of irredundant generators of $G$ is an effective heuristic, since they allow for representing $G$ compactly.

Symmetry-Breaking Answer Set Solving (SBASS) [Drescher et al., 2011] detects and eliminates syntactic symmetries in ASP by adding ground SBCs to an input ground program $P_{g r d}$. A symmetry of $P_{g r d}$ is given by a permutation $\pi$ of ground atoms that that keeps the programs syntactically equivalent, i.e., $P_{g r d}^{\pi}$ has the same rules as $P_{g r d}$, where $P_{g r d}^{\pi}$ is the set of rules obtained by applying $\pi$ to the head and body literals of rules in $P_{g r d}$. In the first step, SBASS transforms $P_{\text {grd }}$ to a colored graph $\mathcal{G}_{P}$ such that permutation groups of $\mathcal{G}_{P}$ and their generators correspond one-to-one to those of $P_{g r d}$. In the second step, it uses SAUCY [Darga et al., 2004; Codenotti et al., 2013] to find a set of group generators

\footnotetext{
${ }^{1}$ It consists of finding all the symmetries of a graph in terms of its generators; it is an attractive problem for the reduction since it can be solved efficiently for many graphs.
} 
for $\mathcal{G}_{P}$. Finally, for each found generator SBASS constructs a set of SBCs and appends them to $P_{g r d}$. Given the modified ground program, an ASP solver can efficiently avoid symmetric answer sets.

\subsection{Inductive Logic Programming}

Inductive Logic Programming [Cropper et al., 2020] is a form of machine learning whose goal is learning a logic program that explains a set of observations in the context of some pre-existing knowledge. Since its foundation, the majority of the research in the topic covers Prolog semantic [Muggleton, 1995; Srinivasan, 2004; Cropper and Muggleton, 2016], even though applications in other logic paradigms appeared in the last years. The most important ILP system for ASP is Inductive Learning of Answer Set Programs (ILASP) [Law et al., 2014; Law et al., 2021], for whom several releases have been developed, extending its learning expressiveness.

The learning task for ILP $\left\langle P, E, H_{M}\right\rangle$ is defined by three elements: a background knowledge $P$, a set of (positive and negative) examples $E$, and a hypothesis space $H_{M}$, which defines all the rules that can be learned. The learned hypothesis is a subset of the hypothesis space that satisfies a specified learning setting: for ILASP, the setting is learning from interpretation [Cropper and Dumančić, 2020]. Before defining it, we need the terminology that ILASP's authors introduced. A Partial Interpretation (PI) is a pair of sets of atoms, $e_{p i}=\langle T, F\rangle$, called inclusions $(T)$ and exclusions $(F)$, respectively. Given a (total) interpretation $\mathcal{I}$ and a PI $e_{p i}$, we say that $\mathcal{I}$ extends $e_{p i}$ if $T \subseteq \mathcal{I}$ and $F \cap \mathcal{I}=\emptyset$. We can augment $e_{p i}$ with an ASP program $C$ to obtain a Context Dependent Partial Interpretation (CDPI) $e=\left\langle e_{p i}, C\right\rangle$. Given a program $P$, a CDPI $\left\langle e_{p i}, C\right\rangle$, and an interpretation $\mathcal{I}$, we say that $\mathcal{I}$ is an accepting answer set of $e$ with respect to $P$ if $\mathcal{I} \in A S(P \cup C)$ such that $\mathcal{I}$ extends $e_{p i}$.

A learning task for ILASP is given by an ASP program $P$ as background knowledge, two sets of CDPIs, $E^{+}$and $E^{-}$, as positive and negative examples, and the hypothesis space $H_{M}$ defined by a language bias $M$, which limits the potentially learnable rules. The learned hypothesis $H \subseteq H_{M}$ must respect the following criteria: (i) for each positive example $e \in E^{+}$, there is some accepting answer set of $e$ with respect to $P \cup H$; and (ii) for any negative example $e \in E^{-}$, there is no accepting answer set of $e$ with respect to $P \cup H$. If multiple hypotheses satisfy the conditions, the system returns one of the shortest. In the article [Law et al., 2018], the authors extended the expressiveness of ILASP, by allowing noisy examples. With this setting, if an example $e$ is not covered (i.e, there is an accepting answer set for $e$ if it is negative, and none if it is positive) then, the corresponding weight is counted as a penalty. Therefore, the learning task becomes an optimization problem with two goals: minimize the length of $H$ and minimize the total penalties for the uncovered examples.

Now, we will define the syntax of ILASP necessary for our work and refer the reader to the system's manual [Law et al., 2021] for further details. A CDPI is expressed as follow:

$$
\text { \#type (ID@W },\{I n C\},\{E x c\},\{C\}) \text {. }
$$

where type is either pos or neg; ID is an unique identifier for the example; $W$ is a positive integer representing the example's weight (if not defined, the weight is infinite); Inc and ExC are two sets of atoms; and C is an ASP program. The language bias can be specified by mode declarations, which define the predicates that may appear in a rule, their argument types and their frequency. Since in our work we aim to learn constraints, we restrict the search space just to rules $r_{i}$ with $H\left(r_{i}\right)=\perp$. To do so, we need to specify only the mode declaration for the body of a rule, expressed by $\#$ modeb $(R, P,(E))$ where $R$ and $E$ are optional and $P$ is a ground atom whose arguments are placeholders of type $\operatorname{var}(t)$ for some constant term $t$. In the learned rules, the placeholders will be replaced by variables of type $t$. The optional element $\mathrm{R}$ is a positive integer called recall which specifies the maximum number of times that the mode declaration can be used in each rule. Lastly, $E$ is a construct, which further restricts the hypothesis search. We limit our interest to the anti reflexive option that works with predicates of arity 2 . Using it, the predicate $P$ should be generated with two distinguished variables.

Choosing an appropriate language bias is still one of the major challenges for modern ILP systems; if the bias does not provide enough limitations, the problem becomes intractable; on the other hand, a strong bias may remove the solution from the search space [Cropper and Dumančić, 2020].

\section{Approach}

We tackle combinatorial problems modeled in ASP such that the instances of a logic program $P$ are generated by a discrete and often stationary stochastic process. Such situations occur, e.g., in industrial settings where the encoding of a manufacturing system is fixed and production orders vary. In this case, every problem instance can be seen as an outcome of the generation process. We assume that any instance $(i)$ specifies the (true) atoms of unary domain predicates $p_{1}, \ldots, p_{k}$ in $P$, where $c_{i}$ is the number of atoms that hold for each $p_{i}$; and (ii) the satisfiability of the instance depends on the number of atoms for each domain predicate, but not on the values of their terms. Thus, without loss of generality, we consider the terms for each $p_{i}$ to be consecutive integers from 1 to $c_{i}$.

Our method exploits instance-specific SBCs on a representative set of instances and utilizes them to generate examples for an ILP task. Solving the task yields first-order constraints that remove symmetries in the analyzed problem instances as much as possible while preserving the instances' satisfiability. We consider two following learning modes: (i) the default mode is cautious by preserving all answer sets that are not filtered out by the ground SBCs; and (ii) the sat mode aims to learn tighter constraints by only guaranteeing that the learned constraints do not eliminate all answer sets for an instance.

To compute the examples, our approach relies on small satisfiable instances (i.e., with a low value for each $c_{j}$ ), subdivided into two parts: $S$ and Gen. Each instance $g \in G e n$ defines a positive example with empty inclusions and exclusions, and $g$ as context. These examples, denoted by $E x_{G e n}$, guarantee that the learned constraints generalize for the target distribution since they force the constraints to preserve some solution for each $g \in G e n$. The instances $i \in S$ are used to obtain positive and negative examples, representing 


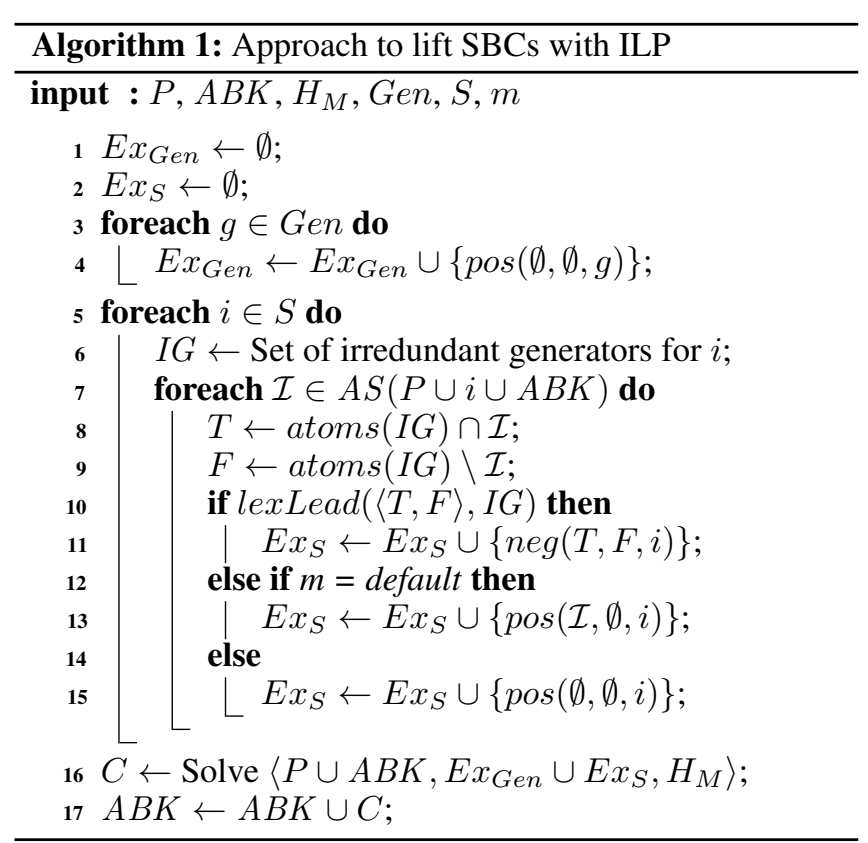

answer sets of $P \cup i$ to be preserved or filtered out, respectively, by corresponding SBCs. We denote their union by $E x_{S}$, where positive examples represent whole answer sets in default mode, or, like instances in Gen, consist of empty inclusions and exclusions along with the context $i$ in sat mode.

An ILP task further requires background knowledge and a hypothesis space $H_{M}$. Both of them are defined by the user (for a possible instantiation, see Section 4.1). The former consists of $P$ along with Active Background Knowledge, denoted by $A B K$ in Algorithm 1, including auxiliary predicate definitions and constraints learned so far. The latter contains the mode declarations, and we assume it to be general enough to entail ground SBCs by learned first-order constraints. The remaining inputs of Algorithm 1 consist of the instances in Gen and $S$ as well as the learning mode $m$. For each answer set $\mathcal{I}$ of an instance $i \in S$ to be analyzed, the predicate lex Lead $(\langle T, F\rangle, I G)$ at line 10 evaluates to true if $\mathcal{I}$ is dominated, i.e., $\mathcal{I}$ can be mapped to a lexicographically smaller, symmetric answer set by means of some irredundant generator in $I G$. In this case, the negative example $\operatorname{neg}(T, F, i)$ is added to $E x_{S}$ in order to eliminate $\mathcal{I}$, while $\operatorname{pos}(\mathcal{I}, \emptyset, i)$ or $\operatorname{pos}(\emptyset, \emptyset, i)$ is taken as the positive example otherwise, depending on whether default or sat mode is selected. Positive examples of the form $\operatorname{pos}(\emptyset, \emptyset, g)$ are also gathered in $E x_{G e n}$ for instances $g \in G e n$, and solving the ILP task at line 16 gives new constraints $C$ to extend $A B K$.

\section{Implementation}

The implementation of our approach relies on CLINGO (consisting of the grounding and solving components GRINGO and CLASP), SBASS and ILASP, and is available at [Tarzariol et al., 2021]. Figure 1 shows the pipeline to generate the examples for a given instance $i \in S$ (the for-loop at line 5 of Algorithm 1). First, $P, i$, and $A B K$ are grounded with GRINGO to get the ground program $P_{\text {grd }}$ in SMODELS format.

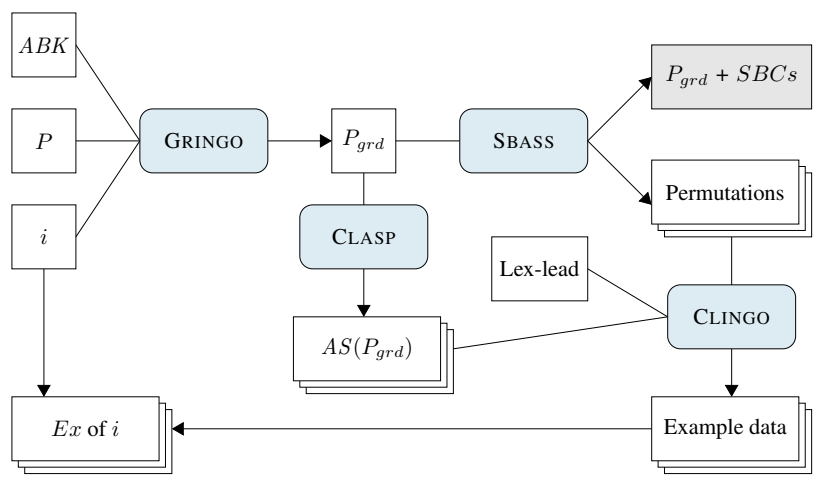

Figure 1: Pipeline to compute examples from instance $i$.

Then, the solver CLASP enumerates all its answer sets, obtaining $A S\left(P_{g r d}\right)$. Independently, SBASS is run on $P_{g r d}$ with the option --show to output the set of permutation group generators. This set contains the vertex permutations of $\mathcal{G}_{P}$, expressed in cycle notation. We extract the cycles defined by vertices representing atoms of $P_{g r d}$ and transform them from SMODELS format back into their original symbolic representation (by a predicate and terms), using the integer values of terms as lexicographic ordering criterion. Next, for efficiency reasons, we partition the permutations into a set $C$ of clusters according to the involved atoms. More precisely, two permutations belong to the same cluster if they share a common atom; otherwise, they are considered separately. For each cluster $c \in C$, we identify the symmetric answer sets in $A S\left(P_{g r d}\right)$ according to $c$, by using an ASP encoding to evaluate SBCs based on the lex-leader predicate given in [Sakallah, 2009]. The encoding returns the undominated atom assignments concerning the atoms in $c$. For each $\mathcal{I} \in A S\left(P_{\text {grd }}\right)$, we check whether an assignment according to $\mathcal{I}$ leads to unsatisfiability. In this case, $\mathcal{I}$ is a symmetric answer set and, therefore, produces a negative example. We assign a unique identifier to each negative example and a weight of 100 . Due to the weights, ILASP returns a set of constraints even if some negative examples are not covered; moreover, we use uniform weights so that all negative examples have the same relevance and as many as possible are to be eliminated. Since we consider several clusters of permutations, the same answer set $\mathcal{I}$ may be symmetric for more than one cluster. If $\mathcal{I}$ is symmetric for $n$ clusters, our system produces $n$ negative examples for it, with a weight of $\left\lceil\frac{100}{n}\right\rceil$ for each. Lastly, answer sets that were not found to be dominated for any of the clusters in $C$ yield positive examples according to the selected mode. Such positive examples are unweighted; thus, the learned hypothesis must cover all of them.

\subsection{ILP Learning Task}

After considering the example generation, we specify components of the ILP learning task that are suitable for the learning of constraints. The idea is to encode the predicates used by lex-leader symmetry breaking to order atoms and extract the maximal values for domain predicates. Since the mode declarations of ILASP (v4.0.0) do not support arithmetic built-ins such as $<$, we provide auxiliary predicates 
in $A B K$ to simulate them. Presupposing the presence of unary domain predicates $p_{1}, \ldots, p_{k}$ with integers from 1 to $c_{i}$ for each $p_{i}, A B K$ defines the auxiliary predicates $\max p_{i}\left(c_{i}\right)$ for each $p_{i}$ and lessThan $\left(t_{1}, t_{2}\right)$ for each pair of integers $1 \leq t_{1}<t_{2} \leq \max \left\{c_{i} \mid i=1, \ldots, k\right\}$. These two predicates are minimal for overcoming limitations of ILASP to learn lexleader SBCs. The selection of small yet representative instances for $S$ and Gen depends on the considered problem. Regarding $S$, we pursued the strategy that empirically determines instances for which SBASS yields a manageable number of permutation group generators. The instances in Gen are usually larger yet still solvable in a short running time to check that the learned constraints generalize.

Our language bias includes \#modeb $\left(2, p_{i}\left(\operatorname{var}\left(t_{i}\right)\right)\right)$ and \#modebo $\left(1, \max p_{i}\left(\operatorname{var}\left(t_{i}\right)\right)\right)$ as mode declarations for each domain predicate $p_{i}$, where $t_{i}$ is a placeholder indicating the domain for which each $p_{i}$ holds. Moreover, for each (non-auxiliary) predicate $\mathrm{P}$ appearing in some of the generators computed for instances in $S$, we use \#modeb $(2, P)$, where the domains of variables in atoms of $\mathrm{P}$ are indicated by a vector of the placeholders in $\left\{t_{i} \mid\right.$ $i=1, \ldots, k\}$, depending on the role of $\mathrm{P}$ in the given program $P$. In addition, we include mode declarations \#modeb $\left(2\right.$, lessThan $\left.\left(\operatorname{var}\left(t_{i}\right), \operatorname{var}\left(t_{j}\right)\right)\right)$ for all $i, j=$ $1, \ldots, k$, with the option anti reflexive in case $i=j$.

Iterative learning. Inspired by the lifelong learning approach [Cropper et al., 2020], we apply our framework incrementally to a split learning task. In every iteration, we exploit the constraints learned so far to tackle the remaining symmetries. To this end, we divide the hypothesis space for programs with three or more types of variables in the language bias: in the first ILP run, the mode declarations are restricted to two types of variables, say $t_{1}$ and $t_{2}$, and then they are progressively extended to further types from $t_{3}$ to $t_{k}$. This iterative approach can speed up the learning procedure and required less than a minute of running time for each iteration on the combinatorial problems investigated in Section 5.

Example. To illustrate a feasible outcome of our ILP approach, let us inspect constraints learned for the pigeon-hole problem, which is about checking whether $p$ pigeons can be placed into $h$ holes such that each hole contains at most one pigeon. We use the following ASP encoding for this problem:

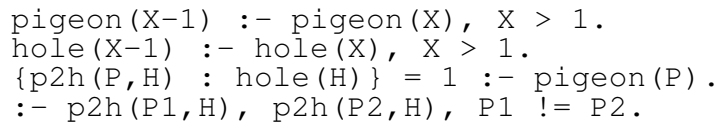

Assume that $S$ consists of the instance with three pigeons and three holes only, which has six answer sets. Analyzing the instance with SBASS gives four generators identifying five symmetric answer sets. These are encoded as negative examples:

\#neg (id1@100, $\{\mathrm{p} 2 \mathrm{~h}(2,3), \mathrm{p} 2 \mathrm{~h}(1,2), \mathrm{p} 2 \mathrm{~h}(3,1)\}$, $\{\mathrm{p} 2 \mathrm{~h}(2,1), \mathrm{p} 2 \mathrm{~h}(1,1), \mathrm{p} 2 \mathrm{~h}(3,3), \mathrm{p} 2 \mathrm{~h}(1,3)$,

p2h $(3,2)$, p2h $(2,2)\}, \quad\{$ pigeon $(3)$. hole $(3)\}$.$) .$ \#neg (id3@100, $\{\mathrm{p} 2 \mathrm{~h}(2,1), \operatorname{p} 2 \mathrm{~h}(3,2), \mathrm{p} 2 \mathrm{~h}(1,3)\}$, $\{\mathrm{p} 2 \mathrm{~h}(1,1), \mathrm{p} 2 \mathrm{~h}(3,3), \mathrm{p} 2 \mathrm{~h}(3,1), \mathrm{p} 2 \mathrm{~h}(2,2)$,

p2h $(2,3)$, p2h $(1,2)\}, \quad\{$ pigeon $(3)$. hole (3). $\})$. \#neg (id4@100, $\{\mathrm{p} 2 \mathrm{~h}(2,3), \mathrm{p} 2 \mathrm{~h}(1,1), \mathrm{p} 2 \mathrm{~h}(3,2)\}$, $\{\mathrm{p} 2 \mathrm{~h}(2,1), \mathrm{p} 2 \mathrm{~h}(3,3), \mathrm{p} 2 \mathrm{~h}(3,1), \mathrm{p} 2 \mathrm{~h}(1,3)$,

p2h $(2,2)$, p2h $(1,2)\}, \quad\{p i g e o n(3)$. hole (3). \}). \#neg (id5@100, $\{\mathrm{p} 2 \mathrm{~h}(2,1), \operatorname{p} 2 \mathrm{~h}(3,3), \operatorname{p} 2 \mathrm{~h}(1,2)\}$, $\{\mathrm{p} 2 \mathrm{~h}(1,1), \mathrm{p} 2 \mathrm{~h}(3,1), \mathrm{p} 2 \mathrm{~h}(1,3), \mathrm{p} 2 \mathrm{~h}(3,2)$, $\mathrm{p} 2 \mathrm{~h}(2,3), \mathrm{p} 2 \mathrm{~h}(2,2)\}, \quad\{$ pigeon $(3)$. hole (3). \}). \#neg (id6@100, \{p2h $(1,1), \operatorname{p} 2 \mathrm{~h}(3,3), \operatorname{p} 2 \mathrm{~h}(2,2)\}$, $\{\mathrm{p} 2 \mathrm{~h}(2,1), \mathrm{p} 2 \mathrm{~h}(3,1), \mathrm{p} 2 \mathrm{~h}(1,3), \mathrm{p} 2 \mathrm{~h}(3,2)$,

$\mathrm{p} 2 \mathrm{~h}(2,3), \mathrm{p} 2 \mathrm{~h}(1,2)\}, \quad\{$ pigeon $(3)$. hole (3). $\})$.

The single positive example in default mode is the following:

\#pos (id2, $\{\mathrm{p} 2 \mathrm{~h}(3,1), \mathrm{p} 2 \mathrm{~h}(2,2), \mathrm{p} 2 \mathrm{~h}(1,3)\}$,

\{\}, pigeon(3). hole (3).\}).

After running ILASP, the learned first-order constraints are:

:- p2h(X,Y), lessThan $(\mathrm{Z}, \mathrm{Y})$, maxpigeon $(\mathrm{X})$.

\% do not assign the pigeon with the max

\% label to a hole other than the first one

:- p2h(X,Y), lessThan $(X, Y)$, lessThan $(Y, Z)$.

\% for all but the last hole, do not assign

o a pigeon with a smaller label to the hole

\section{Experiments}

To evaluate our approach and the implementation design, we applied our framework to a series of combinatorial search problems. For each considered problem, we compared the running time of the original encoding, the version extended with our learned constraints, and the instance-specific approach of SBASS. The learned constraints depend on several aspects, e.g., the selected inputs or whether and how we apply the iterative learning approach. In the following, we report results for the constraints learned applying the definitions of Section $4 .^{2}$ We ran our tests on an Intel ${ }^{\circledR}$ Xeon $^{\circledR}$ E5520 machine under Linux (Ubuntu 18.04.3), where each run was limited to 900 seconds time and 20 GB memory. In Table 1 to Table 4 , the satisfiable instances are shown in grey rows, while the white rows contain unsatisfiable instances. The column BASE refers to CLINGO (v5.4.0) run on the original encoding, while ABK-DEF and ABK-SAT report results for the original encoding augmented with first-order constraints learned in the default or sat mode, respectively. The time required by SBASS to compute ground SBCs is given in the corresponding column, and $\mathrm{CLASP}^{\pi}$ provides the solving time obtained with these ground SBCs. Runs that did not finish within the time limit of 900 seconds are indicated by TO entries.

We first tested the pigeon-hole problem, working without any division and iterative analysis of the language bias, and the two learning modes led to similar constraints. The running time comparison in Table 1 shows that the default and the sat mode of our framework bring about a similar speedup for solving satisfiable as well as unsatisfiable instances. In fact, the vast problem symmetries are cut by the learned firstorder constraints, which is particularly important in case of unsatisfiability, where runs on the original encoding without additional constraints do not finish within the time limit. While SBASS also manages to handle the two smallest instances, the computation of permutation group generators becomes too expensive when the instance size grows, in which case we cannot run CLASP ${ }^{\pi}$ with ground SBCs from SBASS.

Next, we tested two extensions of the pigeon-hole problem, adding color and owner assignments. The pigeon-hole problem with colors associates a color with each pigeon and requires pigeons placed into neighboring holes to be of the same color. The version with colors and owners additionally assigns an owner to each pigeon and imposes the

${ }^{2}$ Detailed settings are provided at [Tarzariol et al., 2021]. 


\begin{tabular}{lrrrrr}
\hline & ABK-DEF & ABK-SAT & BASE & SBASS & CLASP $^{\pi}$ \\
\hline p50-h49 & 0.115 & 0.157 & TO & 54.581 & 2.978 \\
p50-h50 & 0.117 & 0.116 & 0.272 & 54.521 & 2.334 \\
p100-h99 & 1.139 & 1.188 & TO & TO & - \\
p100-h100 & 1.200 & 1.301 & 1.845 & TO & - \\
p200-h199 & 8.648 & 8.443 & TO & TO & - \\
p200-h200 & 8.713 & 9.495 & 16.523 & TO & - \\
p300-h299 & 30.643 & 30.237 & TO & TO & - \\
p300-h300 & 30.510 & 30.333 & 55.606 & TO & - \\
p400-h399 & 72.561 & 72.571 & TO & TO & - \\
p400-h400 & 73.701 & 73.554 & 135.031 & TO & - \\
\hline
\end{tabular}

Table 1: Runtime in seconds for pigeon-hole problem.

\begin{tabular}{lrrrrr}
\hline & ABK-DEF & ABK-SAT & BASE & SBASS & CLASP $^{\pi}$ \\
\hline c1-p12-h11 & 2.643 & 0.005 & TO & 0.758 & 0.018 \\
c1-p52-h52 & 0.339 & 0.223 & 0.342 & 63.749 & 1.622 \\
c2-p12-h12 & 9.512 & 0.012 & TO & 0.085 & TO \\
c2-p52-h53 & 0.783 & 0.437 & TO & 103.843 & TO \\
c3-p12-h13 & 6.442 & 0.021 & TO & 0.239 & TO \\
c3-p52-h54 & 6.892 & 0.972 & 2.814 & 653.216 & TO \\
c4-p12-h14 & 6.229 & 0.023 & TO & 0.675 & TO \\
c4-p52-h55 & 5.130 & 1.579 & TO & 633.469 & TO \\
c5-p12-h15 & 7.928 & 0.043 & TO & 1.076 & TO \\
c5-p52-h56 & 27.226 & 2.383 & 8.447 & TO & - \\
\hline
\end{tabular}

Table 2: Runtime in seconds for pigeon-hole problem with colors.

same constraint as with the colors for owners as well. For the pigeon-hole problem with color assignments, we divided the language bias into two parts: the first limiting to predicates whose atoms exclusively include variables of the types pigeon and hole, while the second part allows variables to be of the type color too. Likewise, the problem version with owners and colors required a third language bias extension to variables of the type owner. For both extensions of the pigeon-hole problem, the first-order constraints learned in default mode turned out to be weaker than those obtained in sat mode, while either kind of constraints helped to improve the search for solutions. Table 2 and Table 3 show similar results: the constraints learned with the sat mode lead to the fastest running times for both satisfiable and unsatisfiable instances. For small unsatisfiable instances, the ground SBCs from SBASS lead to better performance than the constraints learned with the default mode. However, as soon as the color (or owner) dimension grows, the runs of CLASP ${ }^{\pi}$ reach the timeout. This behavior is due to the redundancy of the ground SBCs, which slow down the search instead of facilitating it. For some of the satisfiable instances, finding a solution with the constraints learned in default mode takes longer than with the original encoding alone, but the latter also has timeouts that do not occur with our learned first-order constraints.

Lastly, we applied our framework to the houseconfiguration problem [Friedrich et al., 2011], which consists of assigning $t$ things of $p$ persons to $c$ cabinets, where each cabinet has a capacity limit of two things that must belong to the same owner. The running times in Table 4 exhibit the same trend as observed on the previous problems that our first-order constraints help the search, especially those learned with the sat mode. In some cases, the original encoding is quicker to solve satisfiable instances, but it takes considerably longer for unsatisfiable ones. On the other hand, SBASS brings a moderate speedup for unsatisfiable instances,

\begin{tabular}{lrrrrr}
\hline & ABK-DEF & ABK-SAT & BASE & SBASS & CLASP $^{\pi}$ \\
\hline o1-c3-p12-h13 & 2.327 & 0.015 & TO & 0.200 & TO \\
o1-c3-p52-h54 & 2.818 & 0.858 & 2.760 & 394.272 & TO \\
o2-c3-p12-h13 & 1.865 & 0.017 & TO & 0.395 & TO \\
o2-c3-p52-h54 & 1.463 & 1.294 & TO & 745.101 & TO \\
\hline o3-c1-p12-h13 & 2.335 & 0.014 & TO & 0.320 & TO \\
o3-c1-p52-h54 & 2.619 & 1.151 & 2.732 & 392.267 & TO \\
o4-c4-p12-h14 & 1.270 & 0.041 & TO & 1.238 & TO \\
o4-c4-p52-h55 & 6.316 & 3.036 & 10.041 & TO & - \\
\hline o5-c5-p12-h15 & 0.988 & 0.090 & TO & 2.418 & TO \\
o5-c5-p52-h56 & 20.040 & 5.031 & 18.074 & TO & - \\
\hline
\end{tabular}

Table 3: Runtime in seconds for pigeon-hole problem with colors and owners.

\begin{tabular}{lrrrrr}
\hline & ABK-DEF & ABK-SAT & BASE & SBASS CLASP $^{\pi}$ \\
\hline p2-c6-t13 & 0.723 & 0.042 & 292.056 & 0.092 & 18.672 \\
p2-c80-t160 & 7.861 & 8.498 & 10.274 & TO & - \\
p3-c6-t13 & 0.555 & 0.059 & 344.526 & 0.217 & 102.544 \\
p3-c80-t160 & 21.588 & 22.331 & 29.295 & TO & - \\
p4-c6-t13 & 0.609 & 0.063 & 303.573 & 0.728 & 145.049 \\
p4-c80-t160 & 41.551 & 43.521 & 59.380 & TO & - \\
p5-c6-t13 & 0.705 & 0.072 & 329.670 & 1.174 & 582.101 \\
p5-c80-t160 & 69.009 & 72.756 & 99.911 & TO & - \\
p4-c7-t15 & 18.770 & 0.464 & TO & 1.060 & TO \\
p15-c15-t30 & 9.187 & 10.323 & 6.851 & TO & - \\
\hline
\end{tabular}

Table 4: Runtime in seconds for house-configuration problem.

but its performance suffers a lot when the problem size grows.

\section{Conclusions}

This paper introduces a new method to lift the SBCs of combinatorial problems for a target distribution of instances. Our framework addresses the limitations of common instancespecific approaches, like SBASS, since: (i) the knowledge is transferable, as learned constraints preserve the satisfiability for the considered instance distribution; (ii) the first-order constraints are easier to interpret than ground SBCs; (iii) the SBCs are computed offline, allowing for addressing largescale instances, as shown in our experiments; and (iv) the learned constraints are non-redundant, avoiding performance degradation due to an excessive ground representation size.

In the future, we aim to investigate whether the learning of SBCs can be readily applied or further adapted to advanced industrial problems, such as the Partner Unit Problem [Dodaro et al., 2016], as well as complex combinatorial problems with specific instance distributions, like the identification of Graceful Graphs [Petrie and Smith, 2003]. For such application scenarios, the language bias may be enriched, possibly extending the background knowledge with additional predicates. Moreover, we intend to provide automatic mechanisms to select suitable instances for $S$ and Gen from instance collections, support lifelong learning, and further optimize the grounding and solving efficiency of learned constraints.

\section{Acknowledgments}

This work was partially funded by KWF project 28472 , cms electronics GmbH, FunderMax GmbH, Hirsch Armbänder $\mathrm{GmbH}$, incubed IT GmbH, Infineon Technologies Austria AG, Isovolta AG, Kostwein Holding $\mathrm{GmbH}$, and Privatstiftung Kärntner Sparkasse. We thank the anonymous reviewers for helpful comments. 


\section{References}

[Codenotti et al., 2013] P. Codenotti, H. Katebi, K. Sakallah, and I. Markov. Conflict analysis and branching heuristics in the search for graph automorphisms. In IEEE 25th International Conference on Tools with Artificial Intelligence, pages 907-914. IEEE Computer Society, 2013.

[Cohen et al., 2006] D. Cohen, P. Jeavons, C. Jefferson, K. Petrie, and B. Smith. Symmetry definitions for constraint satisfaction problems. Constraints, 11(2-3):115137, 2006.

[Cropper and Dumančić, 2020] A. Cropper and S. Dumančić. Inductive logic programming at 30: A new introduction. https://arxiv.org/abs/2008.07912, 2020.

[Cropper and Muggleton, 2016] A. Cropper and S. Muggleton. Metagol. https://github.com/metagol/metagol, 2016. Accessed: 2021-05-21.

[Cropper et al., 2020] A. Cropper, S. Dumančić, and S. Muggleton. Turning 30: New ideas in inductive logic programming. In 29th International Joint Conference on Artificial Intelligence, pages 4833-4839. ijcai.org, 2020.

[Darga et al., 2004] P. Darga, H. Katebi, M. Liffiton, I. Markov, and K. Sakallah. Saucy. http://vlsicad.eecs. umich.edu/BK/SAUCY, 2004. Accessed: 2021-05-21.

[Devriendt et al., 2016] J. Devriendt, B. Bogaerts, M. Bruynooghe, and M. Denecker. On local domain symmetry for model expansion. Theory and Practice of Logic Programming, 16(5-6):636-652, 2016.

[Dodaro et al., 2016] C. Dodaro, P. Gasteiger, N. Leone, B. Musitsch, F. Ricca, and K. Schekotihin. Combining answer set programming and domain heuristics for solving hard industrial problems. Theory and Practice of Logic Programming, 16(5-6):653-669, 2016.

[Drescher et al., 2011] C. Drescher, O. Tifrea, and T. Walsh. Symmetry-breaking answer set solving. AI Communications, 24(2):177-194, 2011.

[Erdem et al., 2016] E. Erdem, M. Gelfond, and N. Leone. Applications of ASP. AI Magazine, 37(3):53-68, 2016.

[Falkner et al., 2018] A. Falkner, G. Friedrich, K. Schekotihin, R. Taupe, and E. Teppan. Industrial applications of answer set programming. Künstliche Intelligenz, 32(23):165-176, 2018.

[Friedrich et al., 2011] G. Friedrich, A. Ryabokon, A. Falkner, A. Haselböck, G. Schenner, and H. Schreiner. (Re)configuration using answer set programming. In IJCAI 2011 Workshop on Configuration, pages 17-24. CEUR-WS.org, 2011.

[Gebser et al., 2012] M. Gebser, R. Kaminski, B. Kaufmann, and T. Schaub. Answer Set Solving in Practice. Morgan and Claypool Publishers, 2012.

[Gelfond and Lifschitz, 1991] M. Gelfond and V. Lifschitz. Classical negation in logic programs and disjunctive databases. New Generation Computing, 9:365-385, 1991.

[Law et al., 2014] M. Law, A. Russo, and K. Broda. Inductive learning of answer set programs. In 14th European
Conference on Logics in Artificial Intelligence, pages 311325. Springer-Verlag, 2014.

[Law et al., 2018] M. Law, A. Russo, and K. Broda. Inductive learning of answer set programs from noisy examples. Advances in Cognitive Systems, 7:57-76, 2018.

[Law et al., 2021] M. Law, A. Russo, and K. Broda. Ilasp. http://www.ilasp.com, 2021. Accessed: 2021-05-21.

[Lifschitz, 2019] V. Lifschitz. Answer Set Programming. Springer-Verlag, 2019.

[Margot, 2010] F. Margot. Symmetry in integer linear programming. In 50 Years of Integer Programming 19582008, pages 647-686. Springer-Verlag, 2010.

[Mears et al., 2008] C. Mears, M. García de la Banda, M. Wallace, and B. Demoen. A novel approach for detecting symmetries in CSP models. In 5th International Conference on Integration of $A I$ and OR Techniques in Constraint Programming for Combinatorial Optimization Problems, pages 158-172. Springer-Verlag, 2008.

[Muggleton, 1995] S. Muggleton. Inverse entailment and Progol. New Generation Computing, 13(3-4):245-286, 1995.

[Petrie and Smith, 2003] K. Petrie and B. Smith. Symmetry breaking in graceful graphs. In 9th International Conference on Principles and Practice of Constraint Programming, pages 930-934. Springer-Verlag, 2003.

[Puget, 2005] J. Puget. Automatic detection of variable and value symmetries. In 11th International Conference on Principles and Practice of Constraint Programming, pages 475-489. Springer-Verlag, 2005.

[Sakallah, 2009] K. Sakallah. Symmetry and satisfiability. In Handbook of Satisfiability, pages 289-338. IOS Press, 2009.

[Srinivasan, 2004] A. Srinivasan. The Aleph manual. https://www.cs.ox.ac.uk/activities/programinduction/ Aleph, 2004. Accessed: 2021-05-21.

[Tarzariol et al., 2021] A. Tarzariol, M. Gebser, and K. Schekotihin. ILP symmetry breaking. https://github. com/prosysscience/Symmetry_Breaking_with_ILP, 2021. Accessed: 2021-05-21.

[Walsh, 2012] T. Walsh. Symmetry breaking constraints: Recent results. In 26th AAAI Conference on Artificial Intelligence, pages 2192-2198. AAAI Press, 2012. 